\title{
Epidemiología de la infección por VIH en población de alto riesgo de Cartagena, Colombia
}

\author{
María Cristina Navas ${ }^{1}$, Fernando de la Hoz ${ }^{2}$, Ketty Mendoza ${ }^{1}$, Gabriel Carrasquilla ${ }^{3}$, Jorge Boshell ${ }^{2}$
}

\section{Resumen}

Este estudio describe los factores de riesgo y la prevalencia de infección por el virus de la inmunodeficiencia humana $(\mathrm{VIH})$ en una población de 744 prisioneros, trabajadoras sexuales, hombres homo-bisexuales y marineros en Cartagena, uno de los puertos marítimos y turísticos más importantes sobre el mar Caribe.

Entre noviembre de 1993 y abril de 1995, se realizó un estudio transversal de prevalencia de infección por el VIH y sus factores de riesgo en un grupo de 434 hombres y 310 mujeres que participaron voluntariamente. Las poblaciones fueron captadas en: i) dos cárceles, ii) en la clínica pública de enfermedades de transmisión sexual (ETS), iii) en un bar frecuentado por hombres homosexuales y, iv) en la capitanía del puerto de Cartagena.

La prevalencia de infección por el VIH fue de 2,14\% en la población total. Por grupos, los hombres homo-bisexuales presentaron la más alta prevalencia, 12,3\%; los prisioneros, 2,5\%; las trabajadoras sexuales, $0,7 \%$,y los marineros, $0,5 \%$. Los factores de riesgo que se asociaron significativamente con la infección por VIH fueron la conducta homo-bisexual vs. la conducta heterosexual (razón de prevalencia ( $R P=13$, IC95\%:4,3-34,8) y prácticas de sexo anal ( $R P=7, I C 95 \%: 2,4-19)$. El antecedente de relaciones sexuales con extranjeros se correlacionó en el análisis bivariado, pero, su significancia desapareció en el multivariado.

Los datos confirman que el grupo de hombres homo-bisexuales es aún la población en la cual se concentra el mayor peso de la transmisión del VIH en nuestro país. La prevalencia en trabajadoras sexuales es baja comparada con la encontrada en un estudio reciente realizado en Cali. Se evidencia la necesidad de llegar con mejores mensajes a poblaciones que, por su marginalidad, pueden no estar alertas del riesgo que representa la infección por el VIH.

Palabras clave: prevalencia de VIH, razones de prevalencia, factores de riesgo, conducta sexual.

HIV infection epidemiology in high risk population in Cartagena, Colombia

\section{Abstract}

This study describes the risk factors and the prevalence of HIV infection in a population of 744 prisoners, female prostitutes, homo-bisexual men and sailors in Cartagena, one of the most important tourist harbours on the Caribbean sea.

Between November 1993 and April 1995 a cross sectional study was carried out on the prevalence and risk factors of HIV infection in a group of 434 men and 310 women who participated in voluntary

\footnotetext{
${ }^{1}$ Facultad de Medicina, Universidad de Cartagena, Cartagena, Colombia.

${ }^{2}$ Instituto Nacional de Salud, Santa Fe de Bogotá, D.C., Colombia.

${ }^{3}$ Departamento de Microbiología, Universidad delValle-Fundación FES, Cali, Colombia.

Recibido, junio 17 de 1999; aceptado, septiembre 16 de 1999
} 
testing and came from i) two prisons, ii) the Public Clinic of sexually transmitted diseases (STD) iii) a bar frequented by homosexual men and iv) the harbour at Cartagena. Information was gathered using a standardized questionnaire.

The overall prevalence of HIV infection was $2.14 \%$. Homosexual men had the highest HIV prevalence with $12.3 \%$, while prisoners had $2.5 \%$, female prostitutes $0.7 \%$ and sailors $0.5 \%$. The risk factors associated with HIV infection for all groups were being involved in homo-bisexual behaviour (prevalence ratio $=13 \mathrm{Cl} 95 \%$ 4.8-34.8) and anal sex ( $P R=7 \mathrm{Cl} 95 \%=2.4-19)$. Having sex with foreigners was associated in the bivariate analysis but it vanished in the multivariate analysis.

These data confirm that the homosexual group is still the principal population at risk mainly through anal sex. It is interesting to note that HIV infection in prostitutes was lower than expected from studies in other Colombian cities. Improvement in educational methods to reach people in lower socioeconomical strata is needed to decrease the trends in the HIV epidemic in Colombia among high risk groups such as men having sex with men.

Key words: HIV prevalence, risk factors, prevalence ratio, sexual behavior.

\section{Introducción}

La Organización Mundial de la Salud registró más de 1 '900.000 casos de SIDA hasta noviembre de 1998. El número estimado de asintomáticos infectados con el virus de la inmunodeficiencia humana $(\mathrm{VIH}-1)$ se calcula en 33,4 millones de adultos y niños, mientras que 14 millones de personas en todo el mundo han fallecido de causas relacionadas con el VIH. Para Latinoamérica, se estiman en 1,4 millones de individuos infectados con el VIH (1).

Esta región se puede clasificar en dos grupos de acuerdo con la la tasa de prevalencia del adulto: el primer grupo con una prevalencia de 0,5 a $2,0 \%$, el cual incluye países como Perú, Brasil, Venezue-la, Argentina y Honduras, mientras que el segundo grupo comprende países como México, Chile, Ecuador y Colombia, donde la prevalencia se sitúa entre 0,13 y $0,5 \%$ (2).

En Colombia, desde 1983 hasta junio de 1998, se han notificado 19.907 casos de infección por $\mathrm{VIH}$ al sistema de vigilancia del Ministerio de Salud, de los cuales, 11.552 corresponden a individuos asintomáticos y 8.617 a personas que han desarrollado SIDA (3). La mortalidad acumulada de SIDA en Colombia representa el $40 \%$ de los casos de infección porVIH reportados y corresponde a 3.341 muertes (4). Como es de suponer, los datos oficiales del programa colombiano son un subregistro de la situacion real de la epidemia. A través de diversas estrategias se ha estimado que el número de personas infectadas en el país está entre 80.000 y 120.000 , siendo la mayoría individuos de 15 a 49 años, grupo de edad donde se estima que 4 de cada 1.000 están infectados (3).

Aunque la mayor proporción de casos de $\mathrm{VIH} /$ SIDA se siguen presentando en la población masculina, la relación hombre-mujer ha mostrado una marcada disminución como lo muestran los datos de $1986,55: 1$ y de 1994, 8:1. Esto sugiere un aumento de la transmisión heterosexual; sin embargo, hay que ser cauto en el momento de comparar números crudos porque el aumento de los casos femeninos se hace a expensas de una población que es 10 o más veces mayor en número que la población de hombres homosexuales (3-5).

El principal objetivo del presente estudio fue la obtención de información sobre el nivel actual de prevalencia de infección porVIH en población de alto riesgo heterosexual (trabajadoras sexuales), población de alto riesgo homo-bisexual (presos y homosexuales), así como poblaciones de riesgo desconocido (marineros).

El estudio seroepidemiológico en población con conductas de riesgo, representa una forma de estimar la prevalencia actual de la infección por VIH/SIDA, logrando identificar los grupos de mayor prevalencia y el patrón epidemiológico 
predominante, para así poder instaurar o mejorar programas de seguimiento y educación en esas comunidades.

\section{Materiales y métodos}

Entre noviembre de 1993 y abril de 1995, se realizó un estudio de seroprevalencia y factores de riesgo en un grupo de 744 individuos asintomáticos que no conocían su estado serológico para la infección por el VIH. Un total de 434 hombres $(58,3 \%)$ y 310 mujeres $(41,7 \%)$ participaron en forma voluntaria en el estudio, captados en dos prisiones ubicadas en el perímetro urbano de la ciudad, la Clínica Pública de Enfermedades de Transmisión Sexual (ETS), la capitanía del puerto y un bar gay de la ciudad de Cartagena.

Se realizaron charlas de prevención con los diferentes grupos como inducción al estudio. Los participantes accedieron en forma voluntaria a la toma de muestra de sangre y al diligenciamiento de la encuesta de factores de riesgo que incluyó antecedentes y características de la vida sexual, antecedentes de uso de drogas psicoactivas y antecedentes infecciosos, entre otros.

La población total de reclusos de los dos centros penitenciarios era de 684 en el momento del estudio. Se recolectó un total de 196 muestras, 27 mujeres y 169 hombres, que corresponden a $80 \%$ de la población femenina y $26 \%$ de la población masculina.

Una población aproximada de 500 mujeres carnetizadas acudió a la visita semanal obligatoria en la clínica de ETS, con el fin de obtener el permiso de trabajo, durante el período de estudio. Este grupo incluyó trabajadoras sexuales que tenían como lugar de trabajo 16 bares ubicados en diferentes puntos de la ciudad, el puerto marítimo, barcos o ambulantes. Doscientas ochenta y tres trabajadoras sexuales (56\%) participaron en el estudio.

Doscientos marineros fueron captados a través de la capitanía del puerto. La población total de marineros que trabajan en el puerto de Cartagena es desconocida.
Se recolectaron 65 muestras $(32,5 \%)$ de hombres homosexuales y bisexuales en un bar gay de la ciudad, donde asisten alrededor de 200 hombres homo-bisexuales durante el fin de semana.

Las muestras de plasma fueron analizadas por el método de ELISA (Uniform // Organon Teknika). Se utilizaron dos pruebas confirmatorias: inmunofluorescencia indirecta (IF) o Western Blot (WB), las cuales se realizaron en el Laboratorio de Virología del Instituto Nacional de Salud. Se consideró un WB positivo cuando los anticuerpos presentes en la muestra reconocieron una proteína correspondiente a la región genómica Env (gp160, gp120, gp41) y una proteína correspondiente a las regiones genómicas Gag (p24) o Pol (p31). Los resultados se confirmaron por ELISA (Clonatec) y Western Blot (Pasteur), en el Laboratorio de Virología, Hospital Bichat-Claude Bernard, París (6).

Se utilizó el programa Epiinfo V.6.01 para la creación de la base de datos. Se calcularon las frecuencias univariadas y se comparó la frecuencia de cada variable entre positivos y negativos para WB. Las diferencias fueron probadas usando las pruebas estadísticas de chi cuadrado $\left(X^{2}\right)$ o la prueba exacta de Fisher según la presentación de datos. La asociación de los factores examinados en la encuesta con el hecho de ser positivo para WB se evaluó en un análisis bivariado con las razones de prevalencia (RP) y sus intervalos de confianza del 95\% (IC95\%). Se realizó un análisis multivariado (regresión logística) con las variables que se asociaban con la infección por VIH en el análisis bivariado. En el modelo final, se incluyeron todos los grupos de estudio.

\section{Resultados}

Características de la población encuestada. El cuadro 1 muestra la distribución de las principales características de cada una de las poblaciones encuestadas.

Reclusos. El promedio de edad de los reclusos fue de 28 años, siendo el de mayor promedio de los cuatro grupos analizados. $73 \%$ de los participantes eran nativos de la Costa Atlántica. Solamente $9,7 \%$ tiene relaciones sexuales con 
otros hombres; sin embargo, 24,7\% admitió haber tenido contactos homosexuales en su adolescencia. El número de compañeros sexuales ocasionales tuvo una mediana de 12 por año. $63,7 \%$ tiene o ha tenido relaciones con trabajadoras sexuales. Este grupo presentó la frecuencia más alta de abuso de substancias adictivas tales como marihuana, bazuco y cocaína; la droga más usada fue la marihuana seguida por el bazuco; sólo un recluso refirió haber utilizado morfina por vía endovenosa. Así mismo, la mayor frecuencia de transfusiones sanguíneas corresponde a este grupo (14\%), debido posiblemente a un alto número de accidentes con arma cortopunzante o de fuego. Sorprendentemente, el consumo de alcohol fue similar en todos los grupos. La frecuencia de ETS como sífilis y gonorrea fue muy alta; casi la mitad de los encuestados refería haber tenido, po lo menos, un episodio de una ETS en el pasado. La prevalencia del uso de condón fue la más baja de todas las poblaciones encuestadas.

Trabajadoras sexuales. El promedio de edad de la población de trabajadoras sexuales (TS) fue de 25 años, con un rango de 15 a 47 años.

Cuadro 1. Distribución de características y factores de riesgo de la infección por VIH en cuatro grupos de población de alto riesgo en Cartagena, 1993-1995.

\begin{tabular}{|c|c|c|c|c|}
\hline Variables & $\begin{array}{l}\text { Trabajadoras sexuales } \\
\qquad n=283\end{array}$ & $\begin{array}{l}\text { Marineros } \\
\mathrm{N}=200\end{array}$ & $\begin{array}{c}\text { Reclusos } \\
n=196\end{array}$ & $\begin{array}{l}\text { Homo-Bisexuales } \\
\quad n=65\end{array}$ \\
\hline \multicolumn{5}{|l|}{ Edad } \\
\hline 15-21 años & $80(28,3 \%)$ & $33(18 \%)$ & $33(17,2 \%)$ & $27(44,3 \%)$ \\
\hline $22-25$ & $89(31,4 \%)$ & $49(27 \%)$ & $33(17,2 \%)$ & $16(26,2 \%)$ \\
\hline $26-30$ & $62(21,9 \%)$ & $43(24 \%)$ & $53(27,6 \%)$ & $12(19,7 \%)$ \\
\hline$>31$ & $52(18,4 \%)$ & $52(29 \%)$ & $73(38,1 \%)$ & $6(9,8 \%)$ \\
\hline Heterosexual & $283(100 \%)$ & $200(100 \%)$ & $177(90,3 \%)$ & \\
\hline Bisexual & & & $17(8,7 \%)$ & $35(53,8 \%)$ \\
\hline Homosexual & & & $2(1 \%)$ & $30(46,2 \%)$ \\
\hline Antecedente relaciones & & $1(0,5 \%)$ & $48(24,7 \%)$ & \\
\hline \multicolumn{5}{|l|}{ Homosexuales } \\
\hline Sexo con extranjeros & $203(72 \%)$ & $51(25 \%)$ & $27(13,9 \%)$ & $13(20 \%)$ \\
\hline Sexo en el extranjero & $33(11,7 \%)$ & $70(35 \%)$ & $26(13,5 \%)$ & $4(6,3 \%)$ \\
\hline \multicolumn{5}{|l|}{ Parejas ocasionales } \\
\hline 0 & & $81(40,7 \%)$ & $39(26 \%)$ & $8(13,1 \%)$ \\
\hline $1-5$ & & $54(27,1 \%)$ & $21(14 \%)$ & $8(13,1 \%)$ \\
\hline$>6$ & & $64(32,2 \%)$ & $90(60 \%)$ & $45(73,8 \%)$ \\
\hline \multicolumn{5}{|c|}{ Relaciones sexuales con prostitutas } \\
\hline 0 & & & $162(83,5 \%)$ & $46(36,2 \%)$ \\
\hline 1 & & & $28(14,4 \%)$ & $36(28,3 \%)$ \\
\hline $2-3$ & & & $2(1 \%)$ & $22(17,3 \%)$ \\
\hline$>4$ & & & $2(1 \%)$ & $23(18,1 \%)$ \\
\hline Sexo anal & $39(13,8 \%)$ & $33(18 \%)$ & $46(24,6 \%)$ & $60(92,3 \%)$ \\
\hline Sexo oral & $150(53 \%)$ & $81(44,5 \%)$ & $73(37,6 \%)$ & $47(72,3 \%)$ \\
\hline Cigarrillo & $141(50 \%)$ & $48(24 \%)$ & $122(62,2 \%)$ & $28(43,1 \%)$ \\
\hline Marihuana* & $55(19,4 \%)$ & $1(0,5 \%)$ & $75(37,9 \%)$ & $7(10,8 \%)$ \\
\hline Bazuco & $10(3,5 \%)$ & $1(0,5 \%)$ & $34(17,2 \%)$ & $1(1,5 \%)$ \\
\hline Cocaína* & $34(12,1 \%)$ & $1(0,5 \%)$ & $30(15,2 \%)$ & $8(12,3 \%)$ \\
\hline Drogas $^{*}$ & $70(24,7 \%)$ & $1(0,5 \%)$ & $83(43,2 \%)$ & $10(17,2 \%)$ \\
\hline Licor & $240(84,8 \%)$ & $164(82,4 \%)$ & $160(81,6 \%)$ & $58(89,2 \%)$ \\
\hline Transfusiones & $23(8,1 \%)$ & $13(6,5 \%)$ & $28(14,1 \%)$ & $2(3,5 \%)$ \\
\hline Antecedente de sifilis & $23(8,2 \%)$ & $4(2 \%)$ & $12(6,1 \%)$ & $1(1,5 \%)$ \\
\hline Antecedente de gonorrea & $100(35,3 \%)$ & $31(15,5 \%)$ & $88(44,9 \%)$ & $11(16,9 \%)$ \\
\hline ETS & $120(42,4 \%)$ & $36(18,1 \%)$ & $93(48,4 \%)$ & $13(22,4 \%)$ \\
\hline Uso de condón & $272(96,8 \%)$ & $119(65 \%)$ & $38(25,3 \%)$. & $51(78,5 \%)$ \\
\hline siempre & $117(41,3 \%)$ & $45(22,6 \%)$ & $4(2,1 \%)$ & $18(31 \%)$ \\
\hline mayoría de las veces & $98(34,6 \%)$ & $25(12,6 \%)$ & $2(1,0 \%)$ & $10(17,2 \%)$ \\
\hline algunas veces & $53(18,7 \%)$ & $48(24,1 \%)$ & $17(8,9 \%)$ & $14(24,1 \%)$ \\
\hline
\end{tabular}


La procedencia de este grupo varió con respecto al grupo de reclusos, pues sólo $51 \%$ de las TS eran provenientes de la Costa Atlántica. La mediana del número de clientes por semana fue de 5 , con un rango entre 1 y 20 . El $72 \%$ de las TS han tenido relaciones sexuales con extranjeros. Este grupo exhibía el segundo lugar en el uso de substancias adictivas, siendo la marihuana y la cocaína, la drogas más usadas. La gran mayoría mencionó el uso del condón por parte del cliente, pero, sólo $41,3 \%$ refiere un uso constante. Un número importante declaró antecedentes de ETS, de las cuales la más frecuente era la gonorrea. Hay que resaltar que ésta es la ETS hallada con más frecuencia en todos los grupos encuestados.

Homo-bisexuales. El promedio de edad en este grupo fue de 21 años, el menor de los cuatro grupos. $75 \%$ de los encuestados procedía de la Costa Atlántica. 73,8\% refería más de 6 parejas ocasionales en el último año, lo que representa una frecuencia significativamente mayor comparada con la frecuencia en las otras poblaciones masculinas analizadas (marineros y reclusos). Igualmente, la frecuencia de sexo anal y oral fue mucho mayor que en los otros grupos. Sorprendentemente, la frecuencia de ETS fue baja comparada con la de reclusos y TS, similar a la de los marineros, considerado un grupo de mucho menor riesgo. Esta diferencia puede deberse a la distribución por edad, considerablemente menor que en los otros grupos ( $70 \%$ era menor de 25 años).

Marineros. El promedio de edad de los participantes fue de 27,2 años, con un rango de 16 a 56 años. $41,8 \%$ de la población era proveniente de la Costa Atlántica. La mediana de compañeras ocasionales fue de 1 por año, lo que señala este grupo como el de menor promiscuidad en la población de estudio. El consumo de drogas adictivas informado fue muy bajo comparado con los anteriores, además de la menor prevalencia de ETS y el menor consumo de substancias tóxicas. Ninguno de los participantes refirió relaciones homosexuales, solamente uno de los encuestados admitió contacto homosexual durante su adolescencia, lo que los convierte en el grupo con menor preva- lencia de conducta homosexual en la población masculina analizada.

\section{Prevalencia de infección por VIH y factores de riesgo asociados con la infección}

Reclusos. La prevalencia de infección por $\mathrm{VIH}$ en el grupo de reclusos fue de 2,5\% (IC95\%: 0,3-4,6\%). De las 197 muestras analizada, 8 fueron positivas para ELISA, de las cuales 5 fueron confirmados por IF o Western Blot y las tres restantes presentaron un resultado WB indeterminado. Los factores de riesgo asociados con la infección en este grupo fueron: conducta homo-bisexual contra heterosexual ( $\mathrm{RP}=6,2$, IC95\%: 1,11-34,8), prácticas de sexo anal (RP=12,7, IC95\%: 1,45-110,7) y, aunque no fue estadísticamente significativa, la variable sexo oral también se asoció con la infección por $\mathrm{VIH}$ (RP=6,549, IC95\%: 0,75-57,9) (cuadro 2).

Trabajadoras sexuales. La prevalencia de infección por VIH en el grupo de trabajadoras sexuales fue de 0,7\% (IC95\%: -0,3-1,7\%). De las 283 muestras, 6 presentaron un ELISA positivo, dos fueron confirmadas por WB y las cuatro restantes presentaron un resultado indeterminado. Ninguno de los factores de riesgo se asoció con la infección por VIH en forma estadísticamente significativa, aunque el riesgo de relaciones sexuales con extranjeros fue muy alto. Probablemente, el reducido número de personas positivas le restó poder estadístico a esta asociación.

Homo-bisexuales. Este grupo presentó una prevalencia de infección porVIH de 12\% (IC95\%: 4-20\%). De las 65 muestras analizadas, 8 resultaron positivas para ELISA y confirmadas porWB. Los factores de riesgo asociados en forma estadísticamente significativos a la infección por $\mathrm{VIH}$ en el grupo de homo-bisexuales fueron antecedente de sífilis (PR=9,1, IC95\%: 4,5-18,4) y sexo con extranjeros (PR=4,0, IC95\%: 1,1513,9) (cuadro 2).

Marineros. En el grupo de marineros, la prevalencia de infección por $\mathrm{VIH}$ fue de $0,5 \%$ (IC95\%: -0,4-1,4\%). Del total de marineros estudiados, uno resultó positivo para ELISA, confirmado con WB. Ninguno de los factores de 
Cuadro 2. Factores de riesgo asociados con la infección porVIH por grupo (prisioneros, homo-bisexuales) y en la población total de estudio.

\begin{tabular}{lllll}
\hline Variable & InfecciónVIH & No-infecciónVIH & $\begin{array}{c}\text { Razón de } \\
\text { prevalencia }\end{array}$ & IC 95\% \\
\hline $\begin{array}{l}\text { Prisioneros } \\
\text { Conducta homo-bisexual vs. }\end{array}$ & $\mathrm{n}=5$ & $\mathrm{n}=191$ & & \\
heterosexual & 2 & 17 & 6.2 & 1.1134 .8 \\
Sexo oral & 4 & 70 & 6.59 & $0.75-57.9$ \\
Sexo anal & 4 & 43 & 12.7 & $1.45-110.7$ \\
Homo-bisexuales & $\mathrm{n}=8$ & $\mathrm{n}=57$ & 4.0 & $1.15-13.9$ \\
Sexo con extranjeros & 4 & 9 & 2.2 & $0.4-13.6$ \\
Sexo en el extranjero & 1 & 3 & 2.7 & $0.4-20.3$ \\
Sexo oral & 7 & 0 & 9.1 & $4.5-18.4$ \\
Antecedente sífilis & 1 & $\mathrm{~N}=728$ & 13 & $4.8-34.8$ \\
Población total & $\mathrm{n}=16$ & 75 & 7 & $2.4-19$ \\
Conducta homo-bisexual vs. & 10 & 168 & 1.95 & $0.74-5.2$ \\
heterosexual & 11 & 287 & 2.52 & $0.6-10.7$ \\
Sexo anal & 9 & 38 & & \\
Sexo con extranjeros & 2 & & & \\
Antecedente sífilis & & & & \\
\hline
\end{tabular}

riesgo se asoció con la infección porVIH en forma estadísticamente significativa.

Según el análisis de regresión logística de los cuatro grupos de riesgo, las variables asociadas con la infección por VIH fueron: conducta homobisexual vs. heterosexual (PR=13, IC95\%: 4,8$34,8)$, prácticas de sexo anal (PR=7 IC95\%:2,419,8), sexo con extranjeros ( $P R=1,95, I C 95: 0,74$ $5,2)$ y antecedente de sífilis ( $P R=2,52, I C 95 \%$ : 0,6-10,7). El género masculino también se asoció fuertemente con la posibilidad de infección, mientras que el antecedente de ETS aumentó 5 veces la posibilidad de infección por $\mathrm{VIH}$, pero no alcanzó significado estadístico, $\mathrm{p}=0,07$ (cuadro 3).

Cuadro 3. Asociación entre la infección por el VIH y las variables incluidas en el modelo final de regresión logística.

\begin{tabular}{lll}
\hline Variable & OR & IC95\% \\
\hline Sexo anal & 5.3 & $1.7-16.3$ \\
Sexo con extranjeros & 4.8 & $1.6-14.7$ \\
Antecedente de sífilis & 8.1 & $1.0-68$ \\
Sexo masculino & 8.8 & $1.7-45.8$ \\
Edad>15 años & 0.95 & $0.87-1.04$ \\
Antecedente de ETS & 0.2 & $0.04-1.11$ \\
\hline
\end{tabular}

\section{Discusión}

A pesar del bajo registro de casos de VIH/SIDA en la región Caribe de Colombia, se esperaría una mayor prevalencia, considerando la presencia de puertos y ciudades turísticas importantes que facilitan la exposición de los habitantes a la población extranjera flotante lo cual permite la diseminación del virus. La única información disponible sobre la progresion de la epidemia en la Costa Atlántica son los datos regulares del programa de $\mathrm{VIH} / \mathrm{SIDA}$, ya que esta región no ha participado consistentemente en el estudio de vigilancia centinela que el Ministerio de Salud desarrolla desde 1992. En los registros nacionales, Cartagena presenta una tasa de infección por VIH de 39,8 casos por 100.000 habitantes, que corresponde a un valor 7 veces menor que el promedio nacional (280/100.000) (5). La prevalencia total en la población del país es deconocida; sin embargo, los estudios centinelas de poblaciones de bajo riesgo (mujeres embarazadas) muestran consistentemente que sería inferior a 1/1.000 en la población general.

En 1994, el Ministerio de Salud llevó a cabo un estudio de pruebas voluntarias para VIH en todo el país. La prevalencia reportada de este estudio fue de $0,3 \%$ (390/130.000 participantes), valor que corresponde a la prevalencia de infección por VIH en poblacion de donantes de sangre. En nuestro estudio, la prevalencia de infección por $\mathrm{VIH}$ fue de $2,14 \%$ en una población de 744 
individuos (prisioneros, homo-bisexuales, prostitutas y marineros), que corresponde a una prevalencia 7 veces mayor que los datos del Ministerio de Salud, debido principalmente a las conductas de riesgo de lo de prisioneros fue de $2,5 \%$. El riesgo de infección por el VIH se asoció con la práctica de sexo anal y con las relaciones homosexuales, mientras que otros factores como el uso de drogas endovenosas no parece incidir significativamente en la dinámica de la infección. Esta prevalencia coincide con otros estudios realizados en México (8), Canadá (9), Reino Unido (10) y Francia (11), generalmente asociada al uso de psicoactivos endovenosos en prisiones de Canadá y Francia. Este grupo presentó la mayor prevalencia de antecedentes de ETS $(48,4 \%) y$, adicionalmente, la mayor frecuencia de adicción a drogas estimulantes como marihuana, bazuco y cocaína $(43,2 \%)$. Esto refleja la falta de control de las autoridades del penal sobre el ingreso de substancias tóxicas y sugiere que una estrategia de consejería y educación individual puede ser útil. Aunque la transmisión directa de VIH en drogadictos es por contacto con jeringas contaminadas, también pueden considerarse los efectos de psicoactivos en la conducta del individuo, lo que indirectamente puede incrementar el riesgo de infección (12).

La baja prevalencia del grupo de prostitutas $(0,7 \%)$ coincide con otros estudios epidemiológicos llevados a cabo en Colombia $(13,14)$, Perú (15) y México (8). Ninguno de los factores de riesgo pudo ser asociado con la infección por elVIH, debido, posiblemente, a la baja prevalencia de infección en este grupo, lo cual no permitió tener suficientes casos para un adecuado poder estadístico. Una de las limitaciones en este estudio en torno a la proporción de prostitutas $\mathrm{VIH}+$ en la ciudad, es que el grupo se focaliza en las trabajadoras institucionalizadas y no se lograron incluir aquéllas que no acuden a control. Esto puede introducir un sesgo de selección, dado que las personas que acuden a control de ETS pueden tener factores de riesgo diferentes a los de quienes no acuden. Calero y colaboradores (16) y McCarthy y colaboradores (17) han informado que la prevalencia de $\mathrm{VIH}$ en trabajadoras sexuales no licenciadas puede estar entre 3 y $10 \%$. Llama la atención el elevado uso de condón entre este grupo de mujeres, lo que puede explicar su baja prevalencia, 41,3\%. Puede explicarse, también, por el uso constante de preservativo por parte del cliente; sin embargo, no fue consistente el efecio protector cuando se realizó el análisis estadístico.

En nuestro estudio, los hombres homo-bisexuales presentaron la mayor prevalencia de infección $(12,3 \%)$ entre los cuatro grupos analizados. Esta frecuencia de infección es similar a la informada por otros estudios en Chile (18), Perú (19) y Jamaica (20); sin embargo, es inferior a la prevalencia reportada por Merino y colaboradores en un grupo de 294 hombres homosexuales colombianos (21). El antecedente de relaciones sexuales con extranjeros encontrado en este estudio, también había sido referido por Merino y colaboradores; adicionalmente, encontramos la asociación del antecedente de sífilis. La asociación de riesgo de infección por VIH y relaciones sexuales con extranjeros en nuestro estudio, podría sugerir que la proporción de transmisión autóctona todavía no es muy importante, al menos en esta ciudad.

También es muy importante el hallazgo de asociación de ETS como la sífilis con el riesgo e infección por VIH. En Africa, se ha demostrado que el diagnóstico precoz y el tratamiento adecuado para las ETS disminuye hasta en $50 \%$ el riesgo de infectarse por $\mathrm{VIH}$, así que en estas poblaciones de alto riesgo hay que concientizar a las personas para que acudan periódicamente al control médico, especialmente si tienen algún síntoma relacionado con las ETS (23).

Llama la atención que entre los reclusos y las trabajadoras sexuales se encontrara alrededor de $50 \%$ de WB indeterminados. Las causas de este tipo de inconsistencia en el diagnóstico pueden ser múltiples. Entre los motivos técnicos, se ha documentado la inconsistencia de resultados entre lotes de reactivos para ELISA, lo cual puede llevar a falsos positivos en la prueba. También se ha mencionado que la multiparidad, el antecedente de enfermedades degenerativas mielopoyéticas, las mujeres 
embarazadas, las enfermedades autoinmunes y las transfusiones múltiples pueden ser causa de resultados inconcluyentes en el WB. En nuestro caso, podemos descartar los problemas técnicos en el ELISA como la causa de los WB indeterminados. Todas las pruebas ELISA positivas fueron realizadas por duplicado y las doblemente positivas fueron nuevamente probadas en el Laboratorio de Virología del Instituto Nacional de Salud. Dado que la mayoría de las fallas del WB fueron en mujeres, podría pensarse que el embarazo o la multiparidad estén jugando un papel importante en estos resultados. Por otra parte, entre los reclusos, que tenían la mayor prevalencia de transfusiones, este antecedente puede explicar también los problemas del WB (24-26).

En Colombia y en otros países de Latinoamérica, los primeros casos de infección por el virus del $\mathrm{VIH}$ se dieron predominantemente entre hombres homosexuales que se habían infectado en Estados Unidos, lo cual permitió la introducción y subsecuente diseminación de la epidemia de VIH en la década de 1980 (18-20). La población homosexual ha sido y continúa siendo el grupo central en la diseminación de la infección dentro de la comunidad. La prevalencia de hombres bisexuales en una comunidad es la que determina la rapidez con que se dará la transmisión heterosexual, ya que ellos se infectan a partir de la relación con hombres homosexuales y, luego, lo transmiten a sus parejas heterosexuales. Por ello, las acciones de prevención a través de la educación deberían intensificarse aún más en estos grupos $(21,22)$.

Se deben tener ciertas precauciones al interpretar los hallazgos de nuestro estudio; en primer lugar, la participación fue voluntaria en todos los casos, lo cual puede introducir el llamado sesgo del observador (5). El motivo de participación en el estudio varía considerablemente, por ejemplo, mayor sensibilidad hacia la salud pero menos comportamientos de riesgo o deseo de confirmar el resultado de un diagnóstico positivo previo. No es posible evaluar el efecto de este potencial sesgo en nuestro estudio.

Otro motivo de cautela en la interpretación de los resultados es la forma en que fueron medidas las variables de riesgo, especialmente la práctica de sexo anal y oral, ya que no se especificaba si eran de tipo pasivo o activo. Dado que esto tiene particular importancia para la transmisión, es posible que la falta de asociación de estas variables con la infección porVIH, especialmente sexo oral, estén relacionadas con la forma como fueron medidas y no porque no exista relación (5).

Seis de las muestras positivas para WB se incluyeron en un estudio de caracterización molecular de la región C2V3 del gen env de VIH. EI análisis de las secuencias permitió la identificación de VIH-1, subtipo B, en todas las muestras analizadas (27).

Este es el primer estudio realizado en Colombia que encuentra factores de riesgo asociados a la infección por $\mathrm{VIH}$ en grupos con conductas de riesgo y que puede proponer medidas específicas de control, tal como la mejoría en el diagnóstico y en el tratamiento de ETS, así como aumentar las precauciones con parejas extranjeras ocasionales como una forma de aminorar el curso de la epidemia.

\section{Agradecimientos}

Este estudio fue financiado por la Fundación FES, Cali, Colombia. Los autores agradecen a François Simon (Hôpital Bichat-Claude Bernard, París), Alvaro Moreno, Carmelo Dueñas, Josefina Castelar (Universidad de Cartagena), Sara Argote (Hospital Universitario de Cartagena), William Peña, Alba Alvarado, Gisella Vega (DASALUD, Bolívar), Martha González (INS) y Oscar Orozco (Insituto Nacional de Cancerología).

\section{Referencias}

1. World Health Organization. Weekly Epidemiological Report 1998;73:373-6

2. Joint United Nations Program on VIH/AIDS (UNAIDS), World Health Organization. Report of the global VIH/ AIDS epidemic. June 1998.

3. Ministerio de Salud de Colombia. Estadísticas actuales de la epidemia de infección por el VIH en el país. Documento técnico del Programa de Control de VIH/SIDA y ETS. Mayo de 1999.

4. Rodríguez J, Cortés M. Estimación de la cobertura del sistema de notificación nacional de VIH/SIDA. Ministerio de Salud de Colombia. Boletín Epidemiológico del Programa de Control delVIH/SIDA 1994. 
5. Ministerio de Salud de Colombia. Información epidemiológica sobre la infección VIH/SIDA. Boletín Epidemiológico Nacional 1995;2(julio).

6. Consortium for Retroviruses Serology Standardization. Serological diagnosis of human immunodeficiency virus infection by Western blot testing. JAMA 1988;260:674-9.

7. García R, Céspedes J, Angulo C, Bonilla J. Factores de riesgo para infección por elVIH: cuestionario CAP de la encuesta seroepidemiológica nacional. II Simposio de Virología Clínica de la Universidad de Cartagena y I Simposio Internacional de infección porVIH y coinfección VIH-TB. Cartagena, Colombia, junio de 1995.

8. Guerena-Burgueno F, Benenson AS, Sepúlveda-Amor J. VIH prevalence in selected Tijuana sub-population. Am J Public Health 1991;81:623-5.

9. Dufour A, Alary M, Poulin C et al. Prevalence and risk behaviours for VIH infection among inmates of a provincial prison in Quebec city. AIDS 1996;10:1009-15.

10. Bird AG, Gore SM, Jolliffe DW, Burns SM. Second anonymous VIH surveillance in Saughton Prison, Edinburgh: prisoners give a lead to other heterosexuals on being VIH tested. AIDS 1993;7:1277-9.

11. Rotily M, Galinier-Pujal A, Obadia Y, et al. VIH testing infection and associated risk factors among inmates in south-eastern French prisons. AIDS 1994;8:1341-4.

12. Estebanez P, Fitch K, Nájera R. VIH and female sex workers. Bol Ofic Sanit Panam 1993;115:415-37.

13. García-Bernal R, KlaskalW, Castro J, Zhang G, Baum M. HIV/AIDS surveillance in Colombia: regional differences in epidemic trends. AIDS 1997;11:1297-8.

14. EspitiaV.Seroprevalencia de anticuerpos contra VIH en prostitutas femeninas. Colombia Médica 1990;21:46-9.

15. Golenbock DT, Guerra J, Pfister $\mathbf{H}$, et al. Absence of infection with human immunodeficiency virus in Peruvian female prostitutes. AIDS Res Hum Retroviruses 1988;4: 493-9.

16. Calero D, Blandón LF, Sevilla E. Vigilancia epidemio- lógica de VIH y SIDA en prostitutas de Cali, Colombia. Colombia Médica 1993;23:167-72.

17. McCarthy MC, Wignal FS, Sanchez J, et al. The epidemiology of $\mathrm{VIH}-1$ infection in Perú, 1986-1990. AIDS 1996;10:1141-5.

18. Bernal B, Arroyave J, et al. Seroprevalence of AIDS in male and female prostitutes from Chile. Rev Chil Obstet Ginecol 1989;54:310-3.

19. Cáceres PC, Gotuzzo E, Wignall, Campos M. Sexual behaviour and seroprevalence of $\mathrm{VIH}-1$ in homosexual men from Perú. Bol Ofic Sanit Panam 1991;111:218-30.

20. Figueroa JP, Brathwarte A, Ward E, et al. VIH/AIDS epidemic in Jamaica. AIDS 1995;9:761-8.

21. Merino N, Sánchez RL, Muñoz A, Prada G, García CF, Polk BF. VIH-1, sexual practices, and contact with foreigners in homosexual men in Colombia, South America. J Acquir Immune Syndr 1990;3:330-4.

22. World Health Organization. Weekly Epidemiological Record 1997;72:365-72.

23. Groskurt H, Mosha F, Todd J, et al. Impact of improved treatment of sexually transmitted diseases on VIH infection in rural Tanzania:a randomized controlled trial. Lancet 1995;346:530-6.

24. Schochetman G, George R. AIDS testing. New York: CDC; 1994.

25. Velásquez G. Diagnóstico de la infección por VIH. En: Velásquez G, Gómez RD, editores. SIDA: un enfoque integral. Medellín: CIB; 1992.

26. Hirsh M, Curran J. Human Immunodeficiency Virus. En: Field B, Knipe D, Howley P et al., editors. Field's Virology. Philadelphia: Lippincott-Raven Publishers; 1996.

27. Navas M-C, Letourneur F, Gomas E, Boshell J, Saragosti S. Analysis of the V3 loop sequences from 12 HIV type 1-infected patients from Colombia, South America. AIDS Research and Human Retroviruses 1999;15:1141-4. 